\title{
I ndicadores sociodemográficos e de saúde de idosos portadores e não portadores de diabetes
}

\section{Sociodemográficos indicators and health of elderly who have and do not have diabetes}

\author{
I ndicadores sociodemográficos de la salud de los ancianos portadores \\ y no portadores de diabetes
}

\author{
Darlene Mara dos Santos Tavares', Rosalina Aparecida Partezani Rodrigues"
}

\section{RESUMO}

Com o avanço da idade aumenta a ocorrência do diabetes mellitus. Os serviços de saúde necessitam readequar para atender as especificidades desta demanda populacional; para tanto deverá proceder o diagnóstico de saúde da população adstrita. Objetivos: descrever as características de um grupo de idosos portadores e não portadores de diabetes mellitus, segundo as variáveis sóciodemográficas e de saúde, bem como identificar as principais diferenças entre eles. Participaram do estudo 151 sujeitos, com idades acima de 60 anos, com diabetes mellitus (Grupo 1) e 151 sem a referida doença (Grupo 2), emparelhados segundo sexo e faixa etária. Os dados foram coletados através de instrumento estruturado, no domicílio do idoso, processados no programa EPIINFO vs 6 . Foi realizada análise descritiva e teste $X^{2}(p<$ $0,05)$. Verificou-se maior percentual para o sexo feminino $(73,5 \%)$, com $1^{\circ}$ grau incompleto $(70 \%)$, renda de 1 a 2 salários mínimo. Variáveis que apresentaram diferença significativa entre os dois grupos foram: razão de aposentadoria, uso regular de medicamentos, problemas de saúde, procura por serviços públicos de saúde, atendimento e internação. $\mathrm{O}$ atendimento ao idoso se torna um desafio, por sua demanda crescente nos serviços de saúde frente às doenças crônicas, dentre elas, o diabetes mellitus.

Palavras chave: Enfermagem geriátrica; Idoso; Cuidados de Enfermagem; Diabetes mellitus; Planejamento em saúde.

\footnotetext{
ABSTRACT

With the age advance the occurrence of diabetes mellitus increases. The health services need adequacy to meet the specific demands of this population, for that the diagnosis of health of the people assigned should be made. Objectives: To describe the characteristics of a group of elderly who has diabetes and another
}

group who doesn't have diabetes, according to the socialdemographic and health variable, as well as identify the differences between them. Study participants were 151 subjects, aged above 60 years old with diabetes mellitus (Group 1) and 151 without the disease (Group 2 ), paired by sex and age. Data were collected through structured instrument, at the elderly's home, processed in the program EPIINFO vs 6 . Descriptive analysis was performed and $x 2$ test $(p<0.05)$. There was a higher percentage for females $(73.5 \%)$ with $1^{\circ}$ incomplete grade $(70 \%)$, income from 1 to 2 minimum wage. Variables that showed significant differences between the two groups were: ratio of retirement, regular use of medicines, health problems, search for public health services, care and hospitalization. The care to the elderly becomes a challenge, for its growing demand in the health services front of chronic diseases, including the diabetes mellitus.

Key words: Geriatric Nursing; Elderly; Nursing care; Diabetes mellitus; Health Planning.

\section{RESUMEN}

Con el adelanto de la edad aumenta la incidencia de la diabetes mellitus. Los servicios de salud necesitan de adecuación para satisfacer las exigencias específicas de esta población, por tanto debe hacer el diagnóstico de salud de la población asignada. Objetivos: Describir las características de un grupo de ancianos portadores $y$ otro grupo de no

Enfermeira. Mestre e Doutor em Enfermagem. Professora Adjunto do Departamento de Enfermagem em Educação e Saúde Comunitária do Curso de Graduação em Enfermagem da Universidade Federal do Triângulo Mineiro (UFTM). Av. Afrânio Azevedo, 2063. Bairro Olinda. Uberaba/MG Email: darlenetavares@netsite.com.br

"Enfermeira. Mestre e Doutor em Enfermagem. Professora Titular do Departamento de Enfermagem Geral e Especializada. Escola de Enfermagem de Ribeirão Preto. Universidade de São Paulo. E-mail: rosalina@eerp.usp.br 
portadores de diabetes mellitus, de acuerdo con la variable socialdemografica y de la salud, así como identificar las diferencias entre ellos.Participaron del estudio 151 sujetos, con edad superior a 60 años, con diabetes mellitus (Grupo 1) y 151 sin la enfermedad (Grupo 2), enparejados según sexo y faja etaria. Los datos fueron recolectados por medio de instrumento estructurado, en el hogar de los ancianos, tratados en el programa EPIINFO vs 6 . Fue realizado un análisis descriptivo y test $x 2(p$ $<0,05)$. Hubo un porcentaje mayor para las mujeres $(73,5 \%)$ con $1^{\circ}$ grado incompleto (70\%), renta de 1 a 2 salarios mínimos. Las

\section{NTRODUÇÃO}

A incidência do diabetes mellitus vem aumentando mundialmente. Em 1995 estimouse que $4 \%$ da população adulta mundial era portadora desta doença, tal percentual deverá representar, aproximadamente, 5,4\% em 2025. A maior concentração será nos países em desenvolvimento ${ }^{(1)}$.

No Brasil, atualmente, estima-se que $11 \%$ da população com idade de 40 anos ou mais tem o diagnóstico de diabetes mellitus, com tendência de ampliação conforme eleva-se a faixa etária(1). Assim, verifica-se aumento do coeficiente de mortalidade por diabetes mellitus, com o progredir da idade. Estudo realizado no estado de São Paulo demonstrou acréscimo de 2.300 vezes entre os grupos etários de 0-9 e o de 70 anos e mais de idade $^{(2)}$.

A elevação do diabetes mellitus no idoso pode ter várias causas, dentre elas, a relação do processo de envelhecimento com o metabolismo da glicose. Pesquisas têm mostrado a degradação da insulina, bem como a diminuição da velocidade de remoção, no decorrer do processo de envelhecimento ${ }^{(3-4)}$.

O ser humano apresenta com a idade, diminuição progressiva da tolerância à glicose em jejum e em especial pós prandial. Após os 30-40 anos de idade, estudos realizadas mostraram a ocorrência do aumento no nível basal de glicemia de $1-2 \mathrm{mg} / \mathrm{dl}$ e duas horas pós prandial 8-10 mg/dl, por década de idade. Desta forma, tem-se observado um leve aumento na hemoglobina glicolisada, o que confirma a intolerância aos hidratos de carbono produzidos com o envelhecimento ${ }^{(4)}$. variables que mostraron diferencias significativas entre los dos grupos fueron: relación de la jubilación, el uso regular de medicamentos, problemas de salud, búsqueda de los servicios públicos de salud, la atención y hospitalización. La atención a los anciones se convierte en un desafio por su demanda creciente en los servicios de salud frente a las enfermedades crónicas, entre ellas la diabetes mellitus.

Palabras clave: Enfermería geriátrica; Anciano; Atención de enfermería; Diabetes mellitus; Planificación en Salud.

O aumento da produção hepática basal de glicose, o transtorno na supressão da produção hepática de glicose bem como a captação hepática de glicose, representam outros fatores relacionados ao envelhecimento. A diminuição, tanto da atividade física como da massa magra corporal, e o relativo aumento da porcentagem de tecido adiposo podem contribuir na intolerância à glicose, no envelhecimento ${ }^{(3)}$. Não obstante, é difícil dizer se tais fatos são inerentes ao envelhecimento ou se correspondem a etapas evolutivas do diabetes mellitus $^{(5)}$.

As repercuções nas condições de saúde dos portadores de diabetes mellitus são várias, pois geram complicações crônicas; é um dos principais fatores de risco, associado a hipertensão arterial, o que compromete a qualidade de vida e a inserção social em decorrência da perda de produtividade no trabalho, aposentadoria precoce e mortalidade prematura $^{(1)}$.

Estudo realizado com idosos demonstrou que, em geral, apresentam polimorbidades associada $^{(6)}$. Fato este que pode ser um agravante visto que no decorrer de um estudo multicêntrico, realizado no Brasil, observou-se que, aproximadamente, $50 \%$ dos portadores de diabetes mellitus não conheciam esta condição e que $20 \%$ dos que tinham o diagnóstico da doença não realizavam tratamento ${ }^{(1-2)}$.

Nos serviços de saúde verifica-se o aumento de custos e necessidades de atendimento em locais com maior nível de complexidade. A atenção ao diabetes mellitus, no mundo, apresenta custos diretos de 2,5 a $15 \%$ dos gastos utilizados no setor saúde ${ }^{(1)}$. 
Tanto o aumento da demanda de idosos nos serviços de saúde como a maior ocorrência do diabetes mellitus impõe a necessidade de reorganizar a rede de cuidados progressivos à saúde de maneira a absorver a demanda, ser resolutiva e contribuir na prevenção das complicações decorrentes desta doença.

$\mathrm{O}$ envelhecer com qualidade pressupõe mudanças em vários setores da sociedade, assim como em concepções generalizadas sobre saúde, educação, justiça e direitos sociais. É necessária determinação política para que as instituições possam efetivamente trabalhar em conjunto. No Brasil as políticas que focalizam o idoso e sua família, ainda permanecem como um desafio para a sua implementação, que abarca a precária captação de recursos financeiros para atendimento dessa clientela, além do sistema de informação ser frágil no que tange a análise de condições de vida e de saúde, além da inadequada capacitação de recursos humanos ${ }^{(7)}$.

Os dados evidenciam que no Brasil o envelhecimento populacional tem ocorrido de forma desigual. Aproximadamente $45 \%$ dos idosos residem nos Estados de São Paulo (23\%), Rio de Janeiro (11,5\%) e Minas Gerais $(11,2 \%)^{(8)}$. O município de Uberaba, situado no Estado de Minas Gerais e local de realização desta pesquisa, apresenta população idosa de $10,3 \%{ }^{(9)}$. Tais dados reforçam a necessidade de se desenvolverem pesquisas neste município procurando compreender a dinâmica do seu envelhecimento populacional para a reorganização da atenção aos idosos ${ }^{(10)}$.

Ademais, para a realização do planejamento das ações em saúde, pelo município, é necessária a disponibilidade de informações fidedignas, baseadas em dados primários e secundários.

Os dados ora apresentados, indubitavelmente, mostram a magnitude da problemática que representa o diabetes mellitus em geral e especificamente no idoso e alerta para a necessidade premente de realizar ações em saúde que contribuam para reverter este quadro.

Estudos locoregionais possibilitem construir conhecimento sobre a realidade de atuação dos profissionais de saúde, visto que o Brasil apresenta diversidades regionais. Desta forma, esta investigação tem a finalidade de desvelar situações que contribuirão na formulação de ações e políticas públicas para os idosos portadores de diabetes mellitus, fortalecendo a gestão e melhorando as condições de saúde e de vida desta população.

Considerando a epidemiologia do diabetes mellitus, o envelhecimento populacional, especificamente, os percentuais do município de Uberaba, o crescente consumo de serviços de saúde pelos idosos e a necessidade premente de reorganizar a atenção à saúde desta população, visando atender suas especificidades, foram delineados os objetivos deste estudo.

\section{OBJ ETI VOS}

Descrever as características de um grupo de idosos portadores e não portadores de diabetes mellitus atendidos em três serviços de saúde, do interior de Minas Gerais, segundo as variáveis sóciodemográficas e de saúde e identificar as principais diferenças entre os grupos.

\section{PROCEDI MENTOS METODOLÓGI COS}

A amostra populacional foi constituída a partir do número de atendimentos realizados em todos os serviços públicos de saúde do município de Uberaba, que contam com o médico especialista em endocrinologia.

O estudo contou com 151 sujeitos com diagnóstico de diabetes mellitus, denominado grupo 1 e 151 sem a doença, grupo 2, emparelhados segundo o sexo e as faixas etárias: $60 \mid-65$ anos, $65 \mid-70$ e $70 \mid-75$.

Os critérios de inclusão para o grupo 1: estar em tratamento para diabetes mellitus há cinco anos ou mais; ter entre 60 e 75 anos de idade; ser atendido por endocrinologista em um dos três serviços públicos de saúde; ambos sexos, morar no município e aceitar participar da pesquisa.

Para o grupo 2 os critérios de inclusão foram: apresentar exame de glicemia capilar entre 70 a $110 \mathrm{mg} / \mathrm{dl}^{1}$, com no mínimo de 8 horas de jejum; referir não ter conhecimento de ser portador de diabetes mellitus; ter entre 60 e 75 anos de idade; ambos sexos; morar no município, não morar na mesma casa de um 
sujeito que compôs o grupo de 1 e aceitar participar da pesquisa.

O exame de glicemia capilar não se propõe a fazer diagnóstico, constituindo-se em rastreamento, para efeito desta investigação. Portanto, isso se constitui em limitação deste estudo.

A glicemia capilar foi dosada no aparelho Advantage (Roche). O reagente das tiras de teste foi a glicose dexidrogenase. O sangue capilar foi obtido por punção de polpa digital, utilizando-se lancetas descartáveis. O controle de qualidade do monitor foi realizado sempre que necessário, pelas pesquisadoras, utilizandose tiras reagentes de checagem Advantage e ambas as soluções de controle de glicose Advantage (nível 1 e nível 2).

Para coleta dos dados, após a realização do estudo piloto e as adequações pertinentes, constituiu-se equipe composta por cinco entrevistadores, selecionados entre alunos de graduação em enfermagem, que foram treinados e participavam de reuniões sistemáticas com as pesquisadoras.

Os dados foram coletados por meio de instrumento estruturado, baseando-se no questionário Older Americans Resources and Services (OARS), elaborado pela Duke University ${ }^{(11)}$ e adaptado à realidade brasileira ${ }^{(12)}$. As entrevistas foram conduzidas nos domicílios dos sujeitos que preencheram os critérios de inclusão, tendo duração média de 30 minutos.

As variáveis deste estudo foram: sóciodemográficas (sexo, faixa etária, tempo de diagnóstico, estado civil, número de filhos, escolaridade, aposentadoria) e saúde (atividades da vida diária, morbidades, número de dentes, uso de medicamentos, número de refeições, utilização de serviços de saúde, realização de atividade física).

Antes de iniciar a entrevista foi realizada avaliação cognitiva com o idoso, procurando avaliar as condições do mesmo em responder as questões propostas, quando necessário solicitava-se um informante (pessoa que reside na mesma casa que o entrevistado, e que saiba oferecer informações sobre o idoso). Este estudo contou com a participação de dois informantes substitutos, no grupo 1.
Foi construído banco de dados, no programa EPIINFO vs 6 . Os dados coletados foram processados em microcomputador, por duas pessoas, em dupla entrada. Procedeu-se à consistência dos campos e os inconsistentes foram verificados na entrevista original e realizada sua correção.

Para o tratamento estatístico utilizaram-se medidas descritivas. Calculou-se média e desvio padrão para as variáveis quantitativas que apresentavam distribuição normal, do contrário usou-se mediana e primeiro e terceiro quartil. Para as variáveis qualitativas fez-se a distribuição de freqüência simples. As associações foram estudadas a partir do teste Qui quadrado. Considerou significativo quando $p$ $<0,05$.

Este projeto foi aprovado pelo Comitê de Ética em Pesquisa com Seres Humanos da Universidade Federal do Triângulo Mineiro, protocolo $\mathrm{n}$ - 156 . Os idosos assinaram o termo de consentimento livre e esclarecido antes da coleta dos dados, após as informações necessárias e pertinentes, atendendo a Resolução 196/96 do CNS.

\section{RESULTADOS E DI SCUSSÃO}

\section{Características sociodemográficas}

Do total de 151 sujeitos que constituíram o grupo $1,73,5 \%$ é do sexo feminino. Dentre estes, a faixa etária com maior percentual foi $70 \mid-75$ anos (40,5\%); no sexo masculino, o maior percentual desloca-se para a faixa etária de $65 \mid-70$ anos (40\%).

Ressalta-se que os sujeitos que constituíram o grupo 2 possuem os mesmos resultados apresentados acima, visto que foram emparelhados segundo sexo e faixa etária.

Assim como ocorre na maioria do país, o município de Uberaba possuiu maior percentual de idosas $(56,7 \%)$. Entretanto, este percentual elevado pode estar relacionado a captação da amostra do grupo 1 ter sido realizada no serviço de saúde, que tem maior freqüência de mulheres, que tendem a se cuidar mais ${ }^{(2)}$.

Por outro lado, tem-se vivenciado a feminilização no envelhecimento, especialmente nas faixas etárias mais velhas ${ }^{(8)}$. Este fato deverá incitar a discussão e reflexão para a concretização de ações em saúde e sociais 
direcionadas às necessidades destas mulheres, de forma a contribuir com a sua qualidade de vida e integração social.

Para os idosos portadores de diabetes mellitus, a mediana do tempo de diagnóstico foi 10 anos; primeiro quartil 7 anos e terceiro quartil 17 anos.

As complicações crônicas do diabetes mellitus, comprometem a qualidade de vida e guardam certa relação com o tempo da doença. Estima-se que após 15 anos da doença deverá estar presente a cegueira $(2 \%)$; deficiência visual grave (10\%); algum grau de retinopatia (30 a 45\%), nefropatia (20 a 35\%) e doença cardiovascular (10 a $25 \%)^{(1)}$.

No grupo 1, há maior percentual de idosos morando com companheiros $(48,3 \%)$, do que no grupo $2(42,4 \%)$. O grupo 1 teve média de filhos (média $4,1, \pm 2,8$ ) vivos menor que 0 grupo 2 (média 4,6, $\pm 2,7$ ), apresentando diferença negativa entre as mesmas de 0,12 .

A maioria dos idosos mora com companheiro e possui filhos vivos, fato este que suscita a possibilidade do envolvimento familiar no acompanhamento do diabetes. Como, por exemplo, a capacidade de reconhecer sinais de hipoglicemia, hiperglicemia e prestar atendimento; contribuir no tratamento medicamentoso, caso o idoso apresente dificuldade visual ou faça uso de insulina e não queira auto-aplicar; incentivar o idoso a falar sobre seus sentimentos desagradáveis ou não, em relação à doença e suas complicações.

Por outro lado, o profissional de saúde deve estimular o apoio informal ao idoso, identificando familiares, assim como os problemas que dificultam a aderência ao tratamento.

A maioria dos sujeitos do grupo 1 (72,2\%) e do grupo $2(73,5 \%)$ possuem $1^{\circ}$ grau incompleto. Ocorre diminuição de percentuais conforme aumenta a escolaridade, em ambos os grupos. No Brasil os idosos que possuem de 4 a 8 anos de estudo representam 31,5\% e no Estado de Minas Gerais tal percentual corresponde a $30,7 \%$. Nos últimos dez anos o país vivenciou aumento nos percentuais da escolaridade entre os idosos, principalmente, entre os analfabetos e com um ano de estudo (8). Contudo, tais percentuais necessitam de constante crescimento, pois a escolaridade relaciona-se com o cuidado à saúde, uma vez que o analfabetismo está associado ao risco de deficiência e morte ${ }^{(13)}$ e por outro lado, favorece a melhoria da qualidade de vida, gerando maior bem-estar e longevidade ${ }^{(8)}$.

O rendimento, categorizado em salário mínimo, identificou maior percentual de sujeitos do grupo 1 recebendo até 1 salário mínimo $(58,3 \%)$, enquanto no grupo 2 a maior concentração é na faixa $1-2$ salários mínimos $(61,6 \%)$. Estes percentuais corroboram os dados obtidos para o Brasil $(32,6 \%)$ e para o Estado de Minas Gerais $(36,4 \%)$ que evidenciaram a maioria dos idosos com rendimento de ${ }^{1 / 2} \longrightarrow 1$ salário mínimo ${ }^{(8)}$. Esta situação denota as possíveis dificuldades enfrentadas pelos idosos para conseguirem atender as suas necessidades básicas, bem como suprir o cuidado à sua saúde.

Quanto à profissão atual, no grupo 1 são do $\operatorname{lar}(45,8 \%)$ e não exercem atividades $(35,9 \%) ; \quad$ no grupo 2 estes números representaram $\quad 51,7 \%$ e $22,8 \%$, respectivamente.

No grupo $1,55 \%$ dos sujeitos são aposentados por: tempo de serviço $(24,4 \%)$, idade $(37,8 \%)$ e problema de saúde $(37,8 \%)$. Entre aqueles do grupo 2, $57,1 \%$ se aposentaram por: tempo de serviço $(35,3 \%)$, idade $(48,2 \%)$ e problema de saúde $(16,5 \%)$. Verificou-se que o grupo 1 apresentava, numericamente, mais sujeitos se aposentando por problema de saúde do que no grupo $2\left(\chi^{2}=\right.$ $8,3, p=0,0040$ ).

As questões previdenciárias tem sido um dos temas bastante discutido atualmente. No Brasil 57,1\% dos idosos recebem aposentadoria, $12,1 \%$ pensão e $7,4 \%$ ambos os benefícios. No Estado de Minas Gerais os percentuais são semelhantes, significando $57 \%$, $13,4 \%$ e $7,1 \%$ respectivamente. Enquanto que nos países desenvolvidos, em geral, os idosos ao aposentarem saem do mercado de trabalho, no Brasil esta situação difere. Observou-se que $19,2 \%$ dos idosos, com 60 anos e mais, que haviam aposentado continuavam trabalhando, em outro local. A permanência do idoso no mercado de trabalho pode contribuir para mantê-lo ativo, diminuir a discriminação, a marginalização e o isolamento social, além de contribuir com a renda familiar ${ }^{(8)}$. 


\section{Situação de saúde}

As atividades da vida diária de maior grau de dificuldade no grupo 1 foram: subir e descer escada, cortar unhas dos pés, dirigir condução, uso de transportes, fazer limpeza de casa, dificuldade de sair de casa e tarefas domésticas. Verifica-se que no grupo 2 as atividades referidas foram: subir e descer escada, cortar unhas dos pés e dirigir condução.

Verificou-se que os idosos do grupo 1 apresentaram maior grau de dificuldade para subir e descer escada $(\chi 2=28,5 ; p<0,0001)$; fazer compras diversas $(\chi 2=15,9 ; p<0,0012)$; cortar unhas dos pés $(\chi 2=16,1 ; p<0,0011)$; uso de transporte $(\chi 2=18,0 ; p<0,0004)$; fazer limpeza de casa $(\chi 2=29,9 ; p<0,0001)$; realizar tarefas domésticas $(\chi 2=21,2 ; p<$ 0,0001 ) quando comparado ao grupo 2 .

Estudo epidemiológico realizado com idosos em um município do interior de Minas Gerais apresentou resultados semelhantes: cortar unhas dos pés $(18,93 \%)$; fazer compras diversas $(9,40 \%)$; subir e descer escadas $(4,77 \%)$, medicar-se na hora $(4,12 \%)$ e andar perto de casa $(3,82 \%)^{(10)}$.

A diminuição e/ou perda da capacidade funcional influencia diretamente na qualidade de vida do idoso(14). Nesta perspectiva, o profissional de saúde deve procurar identificar, no cotidiano dos serviços de saúde, as atividades da vida diária que estão comprometidas, visando desenvolver ações em saúde que favoreçam a manutenção da independência.

Os principais problemas de saúde referidos por, aproximadamente, $50 \%$ dos sujeitos, no grupo 1 são: reumatismo, artrite, hipertensão arterial, má circulação, problemas cardíacos, para dormir, de coluna e de visão. No grupo 2 foram: hipertensão arterial, má circulação, problemas cardíacos, problemas de coluna e problemas de visão.

No grupo 1 há maior proporção de idosos com hipertensão arterial $(\chi 2=18,9 ; \mathrm{p}<$ $0,0001)$; obesidade $(\chi 2=18,7 ; p<0,0001)$; problemas para dormir $(\chi 2=7,9 ; p<0,0197)$; catarata $(\chi 2=16,0 ; p<0,0003)$; problema renal $(\chi 2=9,4 ; p<0,0090)$; problemas de visão $(\chi 2=27,0 ; p<0,0001)$ e problemas de audição $\left(\chi^{2}=9,5 ; p<0,0087\right)$ do que os idosos do grupo 2. Destaca-se que os sujeitos do grupo 1 relataram que tais morbidades apresentam maior interferência na vida diária do que aqueles do grupo 2 .

A hipertensão arterial e o diabetes mellitus constituem os principais fatores de risco para as doenças cardiovasculares. Ademais, os idosos podem estar mais susceptíveis à ocorrência de polimorbidades. Para o controle da condição crônica do idoso deve-se utilizar a clínica ampliada, de maneira que seja possível o seguimento da terapêutica, a detecção e intervenção precoce em situações de agravos e de quadros agudizados ${ }^{(15)}$.

O uso regular de medicamentos foi referido por $98,0 \%$ do grupo 1 e por $71,5 \%$ entre aqueles do grupo 2. O grupo 1 possui maior percentual de sujeitos que usam regularmente o medicamento do que os do grupo $2\left(\chi^{2}=39,0, p=<0,0001\right)$. Os medicamentos para o tratamento do diabetes mellitus referidos são: os agentes de sulfoniluréias $(48,5 \%)$, as biguanidas $(27,2 \%)$ e insulina $(24,3 \%)$.

Em decorrência das doenças crônicas os idosos podem fazer uso de vários remédios concomitantemente, fato este que deve ser considerado pelos profissionais de saúde. A polifarmácia pode resultar na interação farmacológica, alterando a absorção, modificando tanto o metabolismo pela indução ou inibição enzimática como a taxa de eliminação(15).

Para obter os medicamentos para diabetes mellitus e outras doenças, $78,6 \%$ dos sujeitos do grupo 1, compram e $21,4 \%$ recebem nos serviços de saúde. Entre os sujeitos do grupo 2, $91,1 \%$ compram e $8,9 \%$ também recebem do serviço de saúde.

Os gastos com medicamentos ficaram acima de R\$20,00 por mês, referidos por $83,6 \%$ dos sujeitos do grupo 1 e $59,7 \%$ dentre os do grupo 2. Para obtenção do medicamento, o valor gasto foi o principal problema relatado, nos dois grupos. Os gastos com medicamentos oneram o orçamento familiar, pois como apresentado anteriormente, os idosos possuem baixa renda individual. Este fato pode levar a não adesão ao tratamento pela dificuldade na aquisição do medicamento.

Tanto no grupo $1(63,6 \%)$ quanto no grupo $2(58,3 \%)$ a maioria é edêntulo. Estudo 
Tavares DMS, Rodrigues RAP. Indicadores sociodemográficos e de saúde de idosos portadores e não portadores de diabetes. Rev. Eletr. Enf. [Internet]. 2008;10(4):906-14. Available from: http://www.fen.ufg.br/revista/v10/n4/v10n4a03. htm.

realizado na região Sudeste apresenta percentuais semelhantes a estes $(65,5 \%)^{(16)}$. $\mathrm{Na}$ referida pesquisa observou que os idosos que possuíam de 1 a 19 dentes apresentaram auto-percepção de saúde bucal pior do que os edêntulos, podendo estar relacionado à qualidade dos dentes que podem levar a dor, insatisfação com a mastigação e estética ${ }^{(16)}$.

Quanto ao número de refeições completas/dia, verificou-se que o grupo 1 tem maior preocupação em se alimentar três vezes ou mais ao dia $(83,1 \%)$, do que o grupo 2 $(66,4 \%)$. Uma das abordagens no tratamento do diabetes mellitus é a dieta fracionada, possivelmente os portadores desta doença receberam esta orientação nos serviços de saúde. O fracionamento das refeições favorece o controle glicêmico e facilita a digestão, que com envelhecimento torna-se mais lenta ${ }^{(17)}$.

A dietoterapia do diabetes mellitus exige abordagem individual e orientações eficazes do manuseio, além da consideração dos aspectos culturais, étnicos e financeiros. Para avaliar a aderência, é mister conhecimentos sobre níveis glicêmicos, da hemoglobina glicolisada, lipídios, pressão sangüínea, peso e qualidade de vida(18). Fatores esses nem sempre disponíveis para a realidade brasileira.

No grupo 1, 48,9\% referiram beber mais de 5 copos/líquido/dia; entre aqueles do grupo 2, a maioria ingere de 3 a 5 copos/líquido/dia $(50,9 \%)$. No grupo 1 os idosos consomem maior quantidade de líquidos quando comparados aos do grupo $2\left(\chi^{2}=21,6, p=\right.$ $<0,0001$ ).

A ingestão diária de líquidos para o idoso deve ser, em média, seis a oito copos por dia. Em geral, o idoso não refere sentir sede, sendo considerado por alguns autores desidratado crônico(18), fato este também percebido nesta investigação.

Verificou maior proporção de sujeitos do grupo 1 procurando os serviços públicos $\left(\chi^{2}=\right.$ 35,3, $\mathrm{p}=<0,0001)$ e sendo internado $\left(\chi^{2}=\right.$ $12,1, p=0,0005)$, nos últimos 12 meses, em relação ao grupo 2. A maior presença no serviço de saúde, do grupo 1 , pode estar relacionada a necessidade de acompanhar o diabetes mellitus e também a maior possibilidade de detecção dos agravos à saúde.
Os motivos para a procura de $60 \%$ dos sujeitos do grupo 1 pelo atendimento ambulatorial foram por: descontrole metabólico do diabetes $(36,9 \%)$ e acompanhamento do diabetes $(10,9 \%)$. A internação ocorreu para $31,5 \%$ dos sujeitos, especialmente, por descontrole metabólico do diabetes $(46,2 \%)$ e cirurgias $(34,6 \%)$. No grupo 2, 39,6\% dos sujeitos buscaram atendimento nos serviços de saúde para: consultas de "rotina" e "preventivas" $(48,6 \%)$ e acompanhamento da hipertensão arterial (16,2\%). Neste grupo 14\% necessitaram de internação, especialmente, devido a problemas cirúrgicos e hipertensão arterial (26,7\% cada um).

Esperava-se, neste estudo, números superiores para a procura por atendimento ambulatorial, já que esses apresentam o diagnóstico de diabetes mellitus e requerem assistência mais direta e contínua. A utilização regular dos serviços de saúde, pela população idosa, pode ser considerada normal. Estudo evidenciou que $35,35 \%$ dos entrevistados haviam procurado, pelo menos uma vez, o atendimento, no período de três meses ${ }^{(19)}$.

Observou-se que os sujeitos do grupo 1 buscam o serviço de saúde em decorrência de doenças já instaladas, ocorrendo o inverso com o grupo 2, que o tem procurado para consultas de "rotina". Em geral, os serviços de saúde têm sido procurados quando da instalação de uma doença ou para realização de ações preventivas, especialmente a vacinação. Nessa perspectiva, espera-se que os sujeitos com diabetes mellitus apresentem maior probabilidade de procurar ao serviço de saúde, contudo deve-se considerar o grau de resolutividade que se está tendo no atendimento. A experiência profissional tem mostrado que, por vezes, os sujeitos têm procurado vários serviços de saúde para conseguir ser atendido e ter seu problema resolvido. Os coordenadores dos serviços de saúde devem viabilizar formas de comunicação com a comunidade, que possibilitem detectar as deficiências no atendimento, com vistas a sua reorganização. Há de se considerar também que o idoso pode depender de um membro familiar para ir ao serviço de saúde, sendo este um fator que pode dificultar seu acesso(20).

Em ambos os grupos, aproximadamente $90 \%$ dos sujeitos consideraram satisfatórios 
tanto o atendimento ambulatorial quanto a internação, apesar disto, observou-se que referem ter problemas com o atendimento.

Existe maior proporção de sujeitos do grupo 1 referindo ter problemas no atendimento do serviço de saúde do que no grupo $2\left(\chi^{2}=\right.$ $18,4, p=<0,0001)$. Os principais problemas, nos dois grupos, em ordem decrescente são: tempo de espera grande, dificuldade de acesso, distância, custo e falta de confiança no atendimento dos serviços de saúde.

Dentre as atividades físicas que praticam destacam-se, no grupo $1(55,6 \%)$ e no 2 $(43,1 \%)$ a caminhada. A prática regular de atividades físicas é essencial para a manutenção de qualidade de vida, principalmente entre os idosos, pois interfere no bem estar psicológico e fisiológico. As atividades físicas estimulam a participação ativa dos idosos, evitando sua acomodação física e psicológica ${ }^{(1)}$. Os profissionais de saúde devem estimular a realização de atividade física entre os idosos, contudo devem considerar: início gradativo; ingesta de líquidos; acesso fácil a carboidrato de fácil absorção, caso tenha hipoglicemia, utilização de calçados e meias adequados e realizarem o exame do pés.

\section{CONCLUSÕES}

A maioria da população deste estudo foi constituída por mulheres $(73,5 \%)$ na faixa etária de $70 \mid-75$ anos (40,5\%). A mediana do tempo de diagnóstico de diabetes mellitus foi de 10 anos. Nos dois grupos os idosos moram com companheiros, tem, aproximadamente, quatro filhos vivos e possuem $1^{\circ}$ grau incompleto. O rendimento é maior entre os sujeitos do grupo 2 ( $1-2$ salários mínimos) do que entre os do grupo 1 ( 1 salário mínimo). Cerca de $50 \%$ dos idosos são aposentados, contudo, no grupo 1 há maior proporção de aposentados por problema de saúde enquanto no grupo 2 foi em decorrência do tempo de serviço.

No grupo 1 há maior grau de dificuldade para realizar as atividades da vida diária, maior ocorrência de doenças, uso de medicamentos, procura pelos serviços públicos de saúde, número de internações e problemas no atendimento do serviço de saúde em relação ao grupo 2. Em ambos os grupos, a maioria compra os medicamentos, sendo o seu valor o principal problema para sua aquisição.

O grupo 1 fraciona mais vezes a alimentação e ingere mais líquidos quando comparado ao grupo 2. Aproximadamente $90 \%$ dos sujeitos consideraram satisfatório o atendimento nos serviços de saúde, porém destacaram os principais problemas: tempo de espera grande, dificuldade de acesso, distância, custo e falta de confiança no atendimento.

\section{REFERÊNCIAS}

1. Ministério da Saúde; Secretaria de Atenção à Saúde, Departamento de Atenção Básica. Diabetes mellitus. Brasília (Brasil): Ministério da Saúde; 2006.

2. Franco LJ. Epidemiologia do diabetes mellitus. In: Lessa I. O adulto brasileiro e as doenças da modernidade. São Paulo: Hucitec/ABRASCO; 1998. p. 123-138.

3. Diaz R. Diabetes en el anciano. INDEN. 1988; 13(1): 17-22.

4. Rios MG. Diabetes en el senescente. Rev. Méd. Chile. 1992; 120(5): 593-601.

5. Torella R, Salvatore T, Spicza R. Is the common presence of glucose intolerance in old age a reliable index for the subsequent ocurrence of lasting hyperglycemia? Acta Diabetol. 1986;23(1):57-61.

6. Tavares DMS, Rodrigues RAP. Situação de saúde do idoso diabético. Arq. Geriatr. Gerontol. 1999; 3(1): 11-17.

7. Motta LB, Aguiar AC. Novas competências profissionais em saúde e o envelhecimento populacional brasileiro: integralidade, interdisciplinaridade e intersetorialidade. Rev Ciência \& Saúde Coletiva. 2007; 12(2):363-72.

8. Ministério do Planejamento, Orçamento e Gestão; Instituto Brasileiro de Geografia e Estatística - IBGE. Informação Demográfica e Socioeconômica número 21. Síntese de Indicadores Sociais: uma análise das condições de vida da população brasileira. Brasília (Brasil): Ministério do Planejamento, Orçamento e Gestão; 2007.

9. Ministério do Planejamento, Orçamento e Gestão; Instituto Brasileiro de Geografia e Estatística - IBGE. Pesquisa Nacional por Amostra de Domicilio. Brasília (Brasil). Ministério do Planejamento, Orçamento e Gestão; 2006 
10. Tavares DMS, Pereira GA, I wamoto HH, Miranzi SCC, Rodrigues LR, Machado ARM. Incapacidade funcional entre idosos residentes em um município do interior de Minas Gerais. Texto Contexto - Enfermagem. 2007; 16(1):329.

11. Duke University Center for tue study of aging and human development (England). Multidimensional functional assessment: the OARS methodology Duke University Center for the Study of Aging and Human Development. Durham (USA): Duke University Center; 1978.

12. Ramos LR. Growing old in São Paulo, Brazil. Assessment of Health status and family support of the elderly of different socio-economic strata living in the community [thesis]. London: London School of Hygiene and Tropical Medicine; 1987.

13. World Health Organization. Envelhecimento ativo: uma política de saúde. Brasília (Brasil): Organização Pan-Americana da Saúde; 2005.

14. Rodrigues RAP. Mulheres em mudança no processo de vida e envelhecer [Livre docência]. Ribeirão Preto: Escola de Enfermagem de Ribeirão Preto, Universidade de São Paulo; 1997.

15. Ministério da Saúde. Secretaria de Atenção à Saúde. Departamento de Atenção Básica. Envelhecimento e saúde da pessoa idosa. Brasília (Brasil): Ministério da Saúde; 2006.

16. Matos DL, Lima-Costa MF. Auto-avaliação da saúde bucal entre adultos e idosos residentes na Região Sudeste: resultados do Projeto SB-Brasil, 2003. Cad. Saúde Pública. 2006; 22(8): 1699- 707.

17. Marucci MFN. Alimentação e hidratação: cuidados específicos e sua relação com o contexto familiar. In: Duarte YAO, Diogo MJD. Atendimento domiciliar: um enfoque gerontológico. São Paulo/Rio de Janeiro/Belo Horizonte: Atheneu; 2000. p. 223-35.

18. Franz M. Terapia nutricional médica. In: Davindson MB. Diabetes mellitus: diagnóstico e tratamento. Rio de Janeiro: Revinter; 2001. p.39-68.

19. Veras RP. País jovem com cabelos brancos: a saúde dos idosos no Brasil. Rio de Janeiro: Relume Dumará; 1994.

20. Carboni RM, Reppetto MA. Uma reflexão sobre a assistência à saúde do idoso no Brasil. Revista Eletrônica de Enfermagem [Internet].
2007 [cited 31 dec 2008];9(1):251-60. Available from: http://www. fen.ufg. br/revista/v9/n1/v9n1a20.htm.

Artigo recebido em 28.02.08.

Aprovado para publicação em 31.12.08. 